\title{
ABSTRACT
}

Meetings of The Keio Medical Society

\section{The Mechanism for the Effect of Chinese Medicine on Strait Episode of Complex Diseases}

\author{
Jing-Yan Han \\ Department of Integration of Chinese and Western Medicine, School of Basic Medical Sciences, \\ Peking University, Beijing, China
}

Complex diseases always involve some strait episode, such as myocardial hypertrophy and fibrosis caused by hypertension, microcirculation dysfunction and injury in heart, brain, lever, and intestine following ischemia and reperfusion, endotoxin induced multiorgan injury, and recovery after intestinal mucosa damage, for which intervention by a single medicine remains unsatisfied in clinic.

Chinese medicine has been applied in clinic for more than two thousand years, which presents as a healthcare system using compound formula preparation, treatment based on syndrome differentiation and individualization thus has advantage in dealing with strait episode of complex diseases. However, the mechanism underlying Chinese medicine is not fully understood up till now.
This report will explain the mechanisms underlying the beneficial role of Chinese medicine in therapy of strait episode of complex diseases based on the resent outcomes in study of aforementioned complex diseases. The speaker, using the theory of blood stasis and activating blood to remove stasis in Chinese medicine, classified microcirculation dysfunction induced by different causes, such as ischemia and reperfusion, stress, LPS, common cold, investigated the pathogenesis and progressing of each of which. Furthermore, the report explored the material basis and mechanism for compound formula of Chinese medicine with potential of tonifying Qi and activating blood, removing heat to cool blood, identified the ingredient in the compound formula responsible for respective effect. (Presented at the 1920th Meeting, June 3, 2016) 